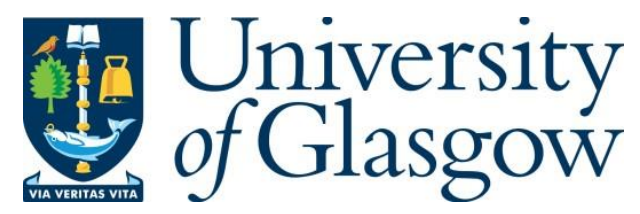

Onireti, O., Mohamed, A., Pervaiz, H. and Imran, M. (2018) A Tractable Approach to Base Station Sleep Mode Power Consumption and Deactivation Latency. In: 2018 IEEE 29th Annual International Symposium on Personal, Indoor, and Mobile Radio Communications (PIMRC) - Workshop WS-04, Bologna, Italy, 09-12 Sep 2018, pp. 123-128. ISBN 9781538660096.

There may be differences between this version and the published version. You are advised to consult the publisher's version if you wish to cite from it.

http://eprints.gla.ac.uk/164326/

Deposited on: 22 June 2018

Enlighten - Research publications by members of the University of Glasgow http://eprints.gla.ac.uk 


\title{
A Tractable Approach to Base Station Sleep Mode Power Consumption and Deactivation Latency
}

\author{
Oluwakayode Onireti*, Abdelrahim Mohamed ${ }^{\dagger}$, Haris Pervaiz ${ }^{\dagger}$ and Muhammad Imran* \\ ${ }^{*}$ School of Engineering, University of Glasgow, Glasgow, UK \\ $\dagger$ Institute for Communication Systems ICS, University of Surrey, Guildford, UK \\ Email: oluwakayode.onireti@glasgow.ac.uk
}

\begin{abstract}
We consider an idealistic scenario where the vacation (no-load) period of a typical base station (BS) is known in advance such that its vacation time can be matched with a sleep depth. The latter is the sum of the deactivation latency, actual sleep period and reactivation latency. Noting that the power consumed during the actual sleep period is a function of the deactivation latency, we derive an accurate closed-form expression for the optimal deactivation latency for deterministic BS vacation time. Further, using this expression, we derive the optimal average power consumption for the case where the vacation time follows a known distribution. Numerical results show that significant power consumption savings can be achieved in the sleep mode by selecting the optimal deactivation latency for each vacation period. Furthermore, our results also show that deactivating the BS hardware is sub-optimal for BS vacation less than a particular threshold value.
\end{abstract}

\section{INTRODUCTION}

A breakdown of the power consumption of the cellular networks reflects that about $80 \%$ of the total power is consumed by the base stations (BSs) [1]. Hence, most optimization effort towards maintaining power consumption evolution in the fifth generation (5G) cellular networks has been on the BS. An accurate power consumption model (PCM) is a prerequisite for such optimization. In [2], the authors presented a linear PCM for the BS in the active state. The PCM for a BS on no-load and sleep mode has been considered in [3]. However, only one sleep mode has been considered in the latter work. In [4], [5], the authors identified four different sleep modes with different power savings based on the BS's hardware capability. Each sleep mode is associated with a given duration; all the BS subcomponents that can enter and exit the sleep mode fast enough are considered sleeping in that mode, while the subcomponents having a longer latency are considered to be still active. In other words, each sleep mode is characterized by a sleep depth (duration) which is the sum of the subcomponent deactivation and reactivation latencies and the actual sleep period. The power saving at each sleep mode is attributed to the actual sleep period [4]. Moreover, the power consumed during subcomponent reactivation and deactivation processes exceeds that of the actual sleep period and in particular,

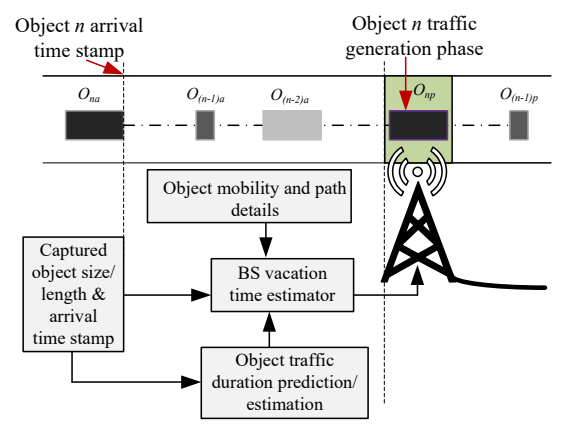

Fig. 1: Estimating the BS vacation and operational time in a single-queue single-server system

the no-load power consumption [6], [7].

The relaxed constraints for BS on/off operation in the control and data separation architecture [8], [9], which is a candidate architecture for $5 \mathrm{G}$ networks [10], means accurate modeling of the sleep mode is essential when evaluating the energy saving gains of this architecture. Accurate evaluation of such gains can only be attained by selecting the optimal sleep depth, i.e., selecting the optimal subcomponent deactivation and reactivation latency for each BS vacation period. The latter refers to the time interval at which the BS is not loaded with data and it is also referred to as the no-load interval. In this paper, we assume that the vacation period of a typical BS is known in advance and that it can be matched with the sleep depth, which minimizes the average power consumption. Furthermore, we consider that the reactivation and deactivation latencies to be proportional. Hence, we propose here the optimal deactivation latency for a BS. Though the knowledge of the vacation is not trivial in a typical cellular network, this assumption is valid for some fixed rate single-queue single-server system such as depicted in Fig. $1{ }^{1}$.

\footnotetext{
${ }^{1}$ In this figure, 1) the time stamp of the object arrival to the system is known, 2) traffic generation takes place some time instance after the object arrival, 3) there exists a known relationship between the generated traffic and object variables such as its length/size/volume, 4) the conditions for traffic generation is pre-defined and known, and 5) the mobility and path details of the object are known. Hence, with some intelligence in the system via a self-organizing network (SON) engine, the vacation and operational time of the BS can be estimated in advance.
} 
In [11], using the time-triggered four sleep modes defined in [4], [5], the authors evaluated the impact of sleeping BS on the overall BS energy consumption. Their results for $4 \mathrm{G}$ showed an energy saving gains up to $22 \%$ due to the limited set of sleep modes available for use. In [12], the authors utilized a curve fitting function to approximate the sleep mode power consumption as a function of the sleep depth. However, little insight could be gained from the analysis due to the high complexity of the approximated function. In this paper, we have followed a tractable approach, which gives more analytical insight, by considering an exponential decay of the actual sleep mode power consumption with subcomponent deactivation latency. Using the developed model, we evaluate the energy saving gain over the discrete four sleep state in [4], [5], [11].

The rest of this paper is organized as follows. We first discuss the network model in Section II, which includes the formulation of the power consumption in the actual sleep phase, the relationship between the reactivation and deactivation latency and, the power consumption in the BS deactivation and reactivation phases. Using this network model in Section III, we accurately obtain the closed-form expression for the deactivation latency that minimizes the average power consumption for a deterministic BS vacation time. Relying on the closed-form expression we provide analytical insights and numerical results in Section IV. Our analysis reveals that deactivating the BS hardware is sub-optimal for BS vacation less than a particular threshold value. Beyond this threshold, increasing the BS vacation time leads to a reduction in the sleep mode power consumption. Conclusions are drawn in Section V.

\section{NETWORK MODEL}

We consider a network where the vacation times of a typical BS are known in advance. In such a network, the $i^{t h}$ vacation time $\tau_{i}$ of a typical BS can be rightly matched with the sleep depth that achieves maximum energy savings. The BS's sleep depth during the $i^{t h}$ vacation period is made up of the component/subcomponent ${ }^{2}$ deactivation latency $\tau_{d i}$, the actual sleep period $\tau_{s i}$, and the subcomponent reactivation latency $\tau_{a i}$, as illustrated in Fig. 2. We consider that the vacation time of a typical BS follows a deterministic or uniform distribution. We aim to obtain the power savings gain that can be achieved as a result of the optimal matching of the sleep depth with the BS vacation time. In order to achieve this, we first present the power consumption at the various phases of the sleep depth.

\section{A. Power Consumption in the Actual Sleep Phase}

We assume that subcomponents deactivation are done such that subcomponents with short deactivation times $^{3}$ are the first to be deactivated. Increasing the BS

\footnotetext{
${ }^{2}$ The terms component and subcomponent are used interchangeably

${ }^{3}$ Note that each subcomponent deactivation time is matched to a corresponding reactivation time
}

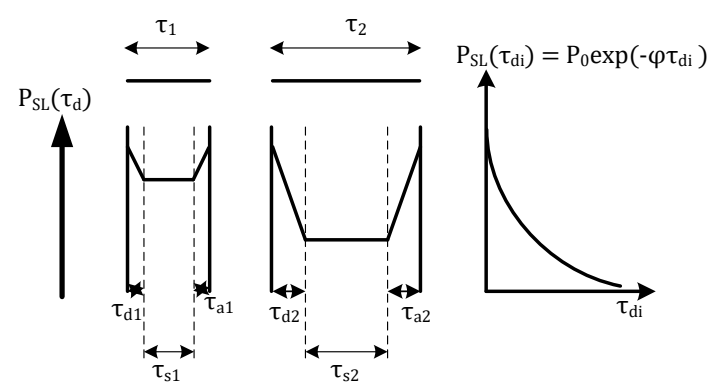

Fig. 2: Base station transition latency showing, subcomponent deactivation latency, actual sleep period and reactivation latency. $P_{S L}$ is the power consumed by the BS during the actual sleep period.

deactivation latency implies deactivating more subcomponents, and hence, a reduction in the power consumed during the actual sleep phase, as illustrated in Fig. 2. This remains the case until when the deepest sleep level, where no further deactivation of subcomponents is possible, has been attained. Hence, in this work, we model the power consumed in the actual sleep state as a function of the deactivation latency. The power consumed at the actual sleep state during the $i^{t h}$ vacation with deactivation latency $\tau_{d i}$ can be modeled as

$$
P_{S L i}\left(\tau_{d i}\right)=\left\{\begin{array}{c}
P_{0} \exp \left(-\psi \tau_{d i}\right) \quad 0 \leq \tau_{d i} \leq \tau_{d, \max } \\
P_{0} \exp \left(-\psi \tau_{d, \max }\right) \quad \tau_{d i}>\tau_{d, \max }
\end{array}\right.
$$

while assuming an exponential decay of the actual sleep mode power consumption with the subcomponent deactivation latency. The parameters $\psi, P_{0}$ and $\tau_{d \text {,max }}$ are the decay constant, no-load power consumption and maximum deactivation latency, respectively.

\section{B. Deactivation and Reactivation Latencies}

Measurement results in [13] have shown that the reactivation latencies of a typical BS's subcomponents always exceed the corresponding deactivation latencies. Hence, we consider that the $i^{t h}$ reactivation latency $\tau_{a i}$ and the corresponding deactivation latency $\tau_{d i}$ are such that

$$
\eta_{i}=\frac{\tau_{a i}}{\tau_{d i}}
$$

where $\eta_{i}>1, \forall i$. Furthermore, for the shortest sleep depth in the long term evolution (LTE) network (71 $\mu \mathrm{s}$ which is equivalent to one OFDM symbol), the small signal blocks considered for deactivation have to show a deactivation latency shorter than one-fourth of the sleepdepth, i.e., $17 \mu \mathrm{s}$ limit defined in [14] for the transmitter transient period [13]. This applies to the reactivation latency as well. By following the same reasoning, we assume that the actual sleep period during the $i^{\text {th }}$ vacation period always exceeds the sum of the deactivation and reactivation latencies, i.e. $\tau_{s i} \geq\left(\tau_{d i}+\tau_{a i}\right)$. Consequently, the deactivation latency is bounded by

$$
\tau_{i} \geq 2 \tau_{d i}\left(1+\eta_{i}\right) .
$$




\section{Deactivation and Reactivation Power Consumption}

The power consumption during subcomponents reactivation is much greater than the no-load power consumption [6], [7]. Hence, following a similar assumption in [15] we model the power consumed during the reactivation phase as

$$
P_{\tau_{a i}}=\theta\left(2 P_{0}-P_{S L_{i}}\left(\tau_{d i}\right)\right)
$$

where $\theta \geq 1$ is a system defined parameter and $P_{0}$ is the no-load power consumption. $P_{\tau_{a i}}$ is a function of the actual sleep period power consumption and it increases with $\tau_{d i}$, since higher $\tau_{d i}$ implies that more subcomponents will be deactivated and hence a higher reactivation power consumption. For the deactivation power consumption $P_{\tau_{d i}}$, we consider it to be a function of the average power consumed over the period of changing from no-load till when the actual sleep level has been attained. Hence, we model $P_{\tau_{d i}}$ as

$$
P_{\tau_{d i}}=\omega\left(P_{0}+P_{S L_{i}}\left(\tau_{d i}\right)\right)
$$

while assuming a linear decrease in power consumption over this period, where $\omega \geq \frac{1}{2}$ is a system defined parameter.

\section{Optimal Average Power Consumption}

\section{A. Average Power Consumption}

Given that the $i^{t h} \mathrm{BS}$ vacation period is matched to a sleep depth, the power consumed during the $i^{t h}$ vacation period, i.e., $\mathcal{E}_{i}$ can be expressed from [15] as follows

$$
\mathcal{E}_{i}=\frac{\tau_{d i} P_{\tau_{d i}}+\tau_{s i} P_{S L i}+\tau_{a i} P_{\tau_{a i}}}{\tau_{i}}
$$

where the first, second and third terms of the numerator are the energy consumed during the BS deactivation, actual sleep and BS reactivation phases, respectively. The terms $P_{S L i}, P_{\tau_{a i}}$ and $P_{\tau_{d i}}$ can be expressed as a function of the subcomponent deactivation latency as in (1), (4) and (5), respectively. Furthermore, the reactivation latency can also be expressed as a function of the deactivation latency as in (2). Given the deterministic vacation period $\tau_{i}$, the actual sleep period $\tau_{s i}$ can also be expressed as a function of the deactivation latency such that

$$
\tau_{s i}=\tau_{i}-\left(\eta_{i}+1\right) \tau_{d i},
$$

where $\eta_{i}=\eta, \forall i$, without loss of generality. Consequently, the average power consumption during the $i^{t h}$ vacation can be further expressed from (6) as

$$
\begin{aligned}
\mathcal{E}_{i}\left(\tau_{d i}, \tau_{i}\right)= & \frac{1}{\tau_{i}}\left(\tau_{d i} P_{S L}\left(\tau_{d i}\right)[\omega-\eta(\theta+1)-1]\right. \\
& \left.+\tau_{i} P_{S L}\left(\tau_{d i}\right)+\tau_{d i} P_{0}(2 \eta \theta+\omega)\right) .
\end{aligned}
$$

\section{B. Optimal Deactivation Latency}

The optimal power consumption during the $i^{t h}$ vacation period can be obtained by finding $\tau_{d i}^{\star}$ that minimizes the power consumption expression in (8). Clearly, $\mathcal{E}_{i}\left(\tau_{d i}\right)$ as defined in (8) is differentiable over its domain, i.e. for $\tau_{d i} \in\left[0, \tau_{d, \max }\right]$, when the actual sleep level $P_{S L}\left(\tau_{d i}\right)$ is as defined in (1) such that $\frac{\partial \mathcal{E}_{i}\left(\tau_{d i}\right)}{\partial \tau_{d i}}$ can be expressed after simplification as

$$
\begin{aligned}
\frac{\partial \mathcal{E}_{i}\left(\tau_{d i}\right)}{\partial \tau_{d i}}=\frac{P_{0}}{\tau_{i}}\left[e^{-\psi \tau_{d i}}\left(Q_{1}-\psi \tau_{i}\right)\right. & -\psi \tau_{d i} Q_{1} e^{-\psi \tau_{d i}} \\
& +2 \eta \theta+\omega],
\end{aligned}
$$

where $\frac{P_{0}}{\tau_{i}}>0$ and $Q_{1}=\omega-\eta(\theta+1)-1$. Let $\tau_{d i}^{\star}$ be the solution the equation $\frac{\partial \mathcal{E}_{i}\left(\tau_{d i}\right)}{\partial \tau_{d i}}=0$. Then $\frac{\partial \mathcal{E}_{i}\left(\tau_{d i}\right)}{\partial \tau_{d i}} \leq 0$ and $\frac{\partial \mathcal{E}_{i}\left(\tau_{d i}\right)}{\partial \tau_{d i}} \geq 0$ for any $\tau_{d i} \in\left[0, \tau_{d i}^{\star}\right]$ and $\left[\tau_{d i}^{\star}, \tau_{d i, \max }\right]$, respectively, which in turn means that $\mathcal{E}_{i}$ decreases over $\tau_{d i} \in\left[0, \tau_{d i}^{\star}\right]$ and increases over $\left[\tau_{d i}^{\star}, \tau_{d i, \max }\right]$. Consequently, for the $i^{t h}$ vacation period, $\mathcal{E}_{i}$ has a unique minimum, which occurs at $\tau_{d i}=\tau_{d i}^{\star}$. Setting $\frac{\partial \mathcal{E}_{i}\left(\tau_{d i}\right)}{\partial \tau_{d i}}=0$ in (9), we obtain that

$$
g\left(\tau_{d i}^{\star}\right)=-(2 \eta \theta+\omega) e^{\psi \tau_{d i}^{\star}}+Q_{1} \psi \tau_{d i}^{\star}=Q_{1}-\psi \tau,
$$

which can be solved in a straightforward manner by means of the Lambert $\mathrm{W}$ function, such that

$$
\begin{aligned}
\tau_{d i}^{\star}=\max \left[\operatorname { m i n } \left[\frac{1}{\psi}(\right.\right. & -W_{0}\left[\frac{-(2 \eta \theta+\omega)}{Q_{1}} e^{\left(1-\frac{\psi \tau_{i}}{Q_{1}}\right)}\right] \\
& \left.\left.\left.+1-\frac{\psi \tau_{i}}{Q_{1}}\right), \tau_{d, \max }\right], 0\right],
\end{aligned}
$$

since $\tau_{d i}^{\star} \in\left[0, \tau_{d, \max }\right]$. Consequently, the optimal power consumption during the $i^{\text {th }}$ vacation can be obtained by substituting $\tau_{d i}^{\star}$ obtained from (11) into (8) such that

$$
\begin{aligned}
\mathcal{E}_{i}\left(\tau_{d i}^{\star}, \tau_{i}\right)= & \frac{1}{\tau_{i}}\left(\tau_{d i}^{\star} P_{S L}\left(\tau_{d i}^{\star}\right)(\omega-\eta(\theta+1)-1)\right. \\
& \left.+\tau P_{S L}\left(\tau_{d i}^{\star}\right)+\tau_{d i}^{\star} P_{0}(2 \eta \theta+\omega)\right) .
\end{aligned}
$$

\section{Optimal Average Power Consumption}

Moreover, given that the vacation time $\tau$ is uniformly distributed in $\left[\tau_{\min }, \tau_{\max }\right]$ such that $f(\tau)=$ $\frac{1}{\tau_{\max }-\tau_{\min }}$, the optimal average power consumption can be expressed as

$$
\begin{aligned}
\mathcal{E}_{A v}^{\star} & =\mathbb{E}_{\tau}\left[\mathcal{E}\left(\tau_{d}^{\star}, \tau\right)\right] \\
& =\frac{1}{\tau_{\max }-\tau_{\min }} \int \mathcal{E}\left(\tau_{d}^{\star}, \tau\right) \mathrm{d} \tau,
\end{aligned}
$$

where $\tau_{d}^{\star}$ is obtained be replacing $\tau_{i}$ with $\tau$ in (11). Since $\tau_{d}^{\star}$ is defined by the max and min operators, we further expand the expression in (13) as

$$
\begin{aligned}
\mathcal{E}_{A v}^{\star}= & \frac{1}{\tau_{\max }-\tau_{\min }}\left(\int_{\widetilde{\tau}_{\min }}^{\widetilde{\tau}} P_{0} \mathrm{~d} \tau+\int_{\widetilde{\tau}}^{\widehat{\tau}} \mathcal{E}\left(\tau, \tau_{d}^{\star}\right) \mathrm{d} \tau\right. \\
& \left.+\int_{\widehat{\tau}}^{\tau_{\max }} \mathcal{E}\left(\tau, \tau_{d, \max }\right) \mathrm{d} \tau\right)
\end{aligned}
$$


where the parameters $\widetilde{\tau}$ and $\widehat{\tau}$ are obtained by solving for $\tau$ in (11) that achieves $\tau_{d}^{\star}=0$ and $\tau_{d}^{\star}=\tau_{d, \max }$, respectively, and $\widetilde{\tau}_{\text {min }}=\min \left(\tau_{\min }, \widetilde{\tau}\right)$. The parameters $\widetilde{\tau}$ and $\widehat{\tau}$ are subsequently defined as

$$
\widetilde{\tau}=\frac{Q_{1}}{\psi}\left(1+\frac{2 \eta \theta+\omega}{Q_{1}}\right)
$$

and

$$
\widehat{\tau}=\frac{Q_{1}}{\psi}\left(1-\psi \tau_{d, \max }+\frac{2 \eta \theta+\omega}{Q_{1}} e^{\psi \tau_{d, \max }}\right),
$$

respectively.

\section{ANALYTICAL InSIGHTS AND NUMERICAL RESULTS}

\section{A. Analytical Insights}

1) Effect of increasing the vacation time $\tau$ on the optimal deactivation latency: Here we consider the scenario where the parameter $\theta=1$ and $\omega=0.5$ in (11). As $Q_{1}=-2 \eta-0.5$ so $\frac{-\psi \tau}{Q_{1}}=\frac{\psi \tau}{2 \eta+0.5}$. We assume that the optimal deactivation latency is unbounded, such that the optimal deactivation latency can be expressed from (11) as

$\tau_{d i}^{\star}=\frac{1}{\psi}\left(-W_{0}\left[\exp \left(1+\frac{\psi \tau}{2 \eta+0.5}\right)\right]+1+\frac{\psi \tau}{2 \eta+0.5}\right)$.

Noting that if $z=W_{0}[z \exp (z)]$, then $z>W_{0}[\exp (z)]$. Consequently, $1+\frac{\psi \tau}{2 \eta+0.5}>W_{0}\left[\exp \left(1+\frac{\psi \tau}{2 \eta+0.5}\right)\right]$ in (17), and $1+\frac{\psi \tau}{2 \eta+0.5}$ dominates the latter expression. Hence, increasing the vacation time leads an increase in the optimal deactivation latency.

2) Effect of increasing the reactivation/deactivation ratio $\eta$ on the optimal deactivation latency: Increasing $\eta$ implies increasing the subcomponent reactivation time. Following the earlier reasoning, it can be observed from (17) that for a fixed vacation time $\tau$, and for $\omega=0.5$ and $\theta=1$, increasing $\eta$ leads to a decrease in the optimal subcomponent deactivation time. This is due to the higher power consumption during the subcomponent reactivation process as compared with the deactivation process.

3) Fixed power consumption in deactivation and reactivation: Given that the power consumption during the subcomponent deactivation and reactivation is fixed to $P_{\tau_{d i}}=P_{\tau_{a i}}=\beta P_{0}$, where $\beta \geq 1$. The unbounded optimal deactivation latency can be expressed as

$$
\tau_{d i}^{\star}=\frac{1}{\psi}\left(-W_{0}\left[\beta \exp \left(1+\frac{\psi \tau}{\eta+1}\right)\right]+1+\frac{\psi \tau}{\eta+1}\right)
$$

after following the same approach as in III-B. It can be seen from (18) that for a fixed $\psi, \eta$ and $\tau$, increasing $\beta$ will lead to a reduction in $\tau_{d i}^{\star}$ and hence deeper sleep level will not be attainable. This implies that research on technologies related to a reduction in the power consumed during the subcomponent deactivation and reactivation processes should be embraced.

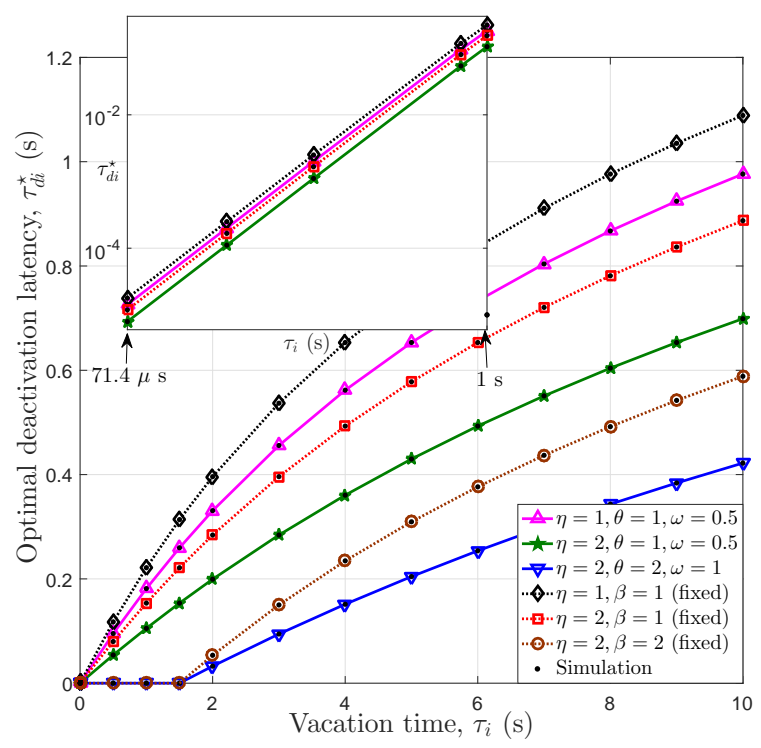

Fig. 3: Optimal deactivation latency as a function of deterministic $\mathrm{BS}$ vacation time.

\section{B. Numerical Results}

In this section, we present some numerical results to illustrate our analytical findings. The system parameters are as follows: $P_{0}=139 \mathrm{~W}[4],[5], \psi=2, \tau_{d i, \max }=$ $2 \mathrm{~s}$.

In Fig. 3, we plot the optimal deactivation latency as a function of deterministic BS vacation time $\tau_{i}$, for $\eta=1,2$, while considering the following scenarios 1 ) the subcomponents reactivation and deactivation power consumption are a function of the attained sleep level as defined in (4) and (5), respectively, 2) the power consumption during the subcomponents deactivation and reactivation processes are fixed to $\beta P_{0}$, where $\beta>1$. Note that the magnified section in the figure shows the optimal deactivation latency for vacation time $[71.4 \mu \mathrm{s}, 1 \mathrm{~ms}, 10 \mathrm{~ms}, 0.5 \mathrm{~s}, 1 \mathrm{~s}]$. It can be seen that for a fixed $\eta$, the optimal deactivation latency increases with the BS vacation time as long as $\tau_{i}>\widetilde{\tau_{i}}$. This observation is intuitive since by increasing the vacation time, the deactivation latency should be increased to allow for the deactivation of more subcomponents and thus reduction in the overall power consumption. This holds as long as the cost of deactivation does not exceed the gains from subcomponent deactivation, as illustrated in Fig. 3 for $\beta=2$ and $\theta=2, \omega=1$. Fig. 3 also shows that increasing $\eta$ leads to a reduction in the optimal deactivation latency. This is due to the higher power consumption during the subcomponent reactivation process as compared to the subcomponent deactivation process. Furthermore, Fig. 3 shows that reducing the power consumed during the subcomponent deactivation and reactivation processes (by reducing $\theta$ and $\omega$ in scenario 1 and reducing $\beta$ in scenario 2) leads to significant increase in the optimal deactivation latency and subsequently increased power savings gain.

In Fig. 4 we plot the average power consumption 


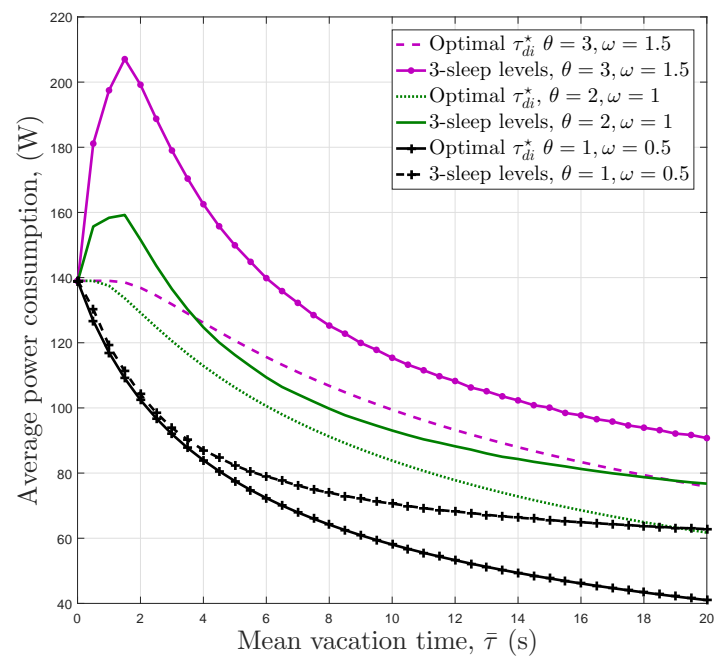

Fig. 4: Average power consumption as a function of the mean vacation time $\bar{\tau}$ for uniformly distributed BS vacation time with $\tau_{\min }=71.4 \mu \mathrm{s}$ and $\tau_{\max }=2 \bar{\tau}-\tau_{\min }$, and $\eta=1$.

against the mean vacation time $\bar{\tau}$, while considering a BS uniformly distributed vacation time with $\tau_{\min }=$ $71.4 \mu \mathrm{s}$ and $\tau_{\max }=2 \bar{\tau}-\tau_{\min }$, and $\eta=1$, for both the case with optimal deactivation latency and the discrete sleep level schemes defined in Table I. In each discrete sleep level, the actual sleep duration of the $i^{t h}$ vacation period can be obtained as $\tau_{s i}=\tau_{i}-(1+\eta) \tau_{d i}^{0}$, where the discrete $\tau_{d i}^{0}$ defined in Table I is dependent on the vacation period. Specifically, we consider that $\tau_{d i}^{0}=0, \forall \tau_{i}<0.5$, while discrete $\tau_{d i}^{0}$ that corresponds to other vacation intervals are stated accordingly in Table I for each discrete sleep level. For the case with $\theta=1, \omega=0.5$, the parameter $\widetilde{\tau}=0$ in (15) and there exists an optimal deactivation latency for each vacation period. Hence, increasing the mean vacation time leads to a reduction in the average power consumption. By increasing the parameters $\theta$ and $\omega$ to 2 and 1 , respectively, and consequently the power consumed during the reactivation and deactivation processes, the parameter $\widetilde{\tau}$ becomes greater than zero in (15) and $\tau_{d i}^{\star}=0, \forall \tau_{i} \leq \widetilde{\tau}$. Hence, in the optimal case, the BS continues to operate in no-load for all vacation time which is less than $\widetilde{\tau}$ since the cost of deactivation and reactivation (increase power during the deactivation and reactivation processes) exceeds the gains from the reduced power consumption due to subcomponent deactivation. This can be seen in Fig. 4 where the average power consumption is equal to $P_{0}$ for $\bar{\tau} \leq 0.5 \mathrm{~s}$ and $\bar{\tau} \leq 1 \mathrm{~s}$ for the optimal case with $\theta=2, \omega=1$ and $\theta=3, \omega=1.5$, respectively. For the suboptimal case with 3-sleep levels, we observe that the average power consumption initially increases with $\bar{\tau}$ to its maximum for $\theta=2, \omega=1$ and $\theta=3, \omega=1.5$, before decreasing with further increase in $\bar{\tau}$. For the suboptimal case, we observe that the average power consumption could even be higher than the no-load power consumption for some

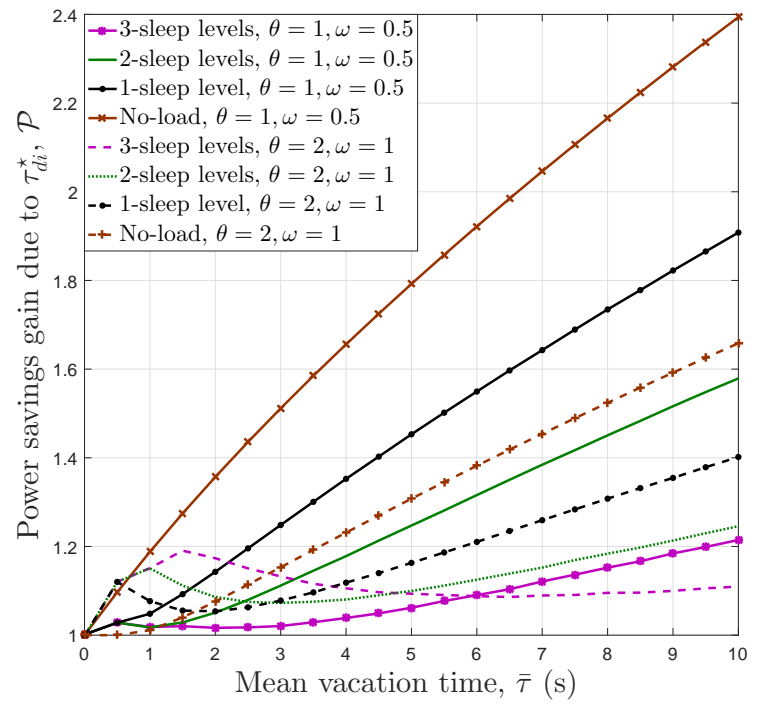

Fig. 5: Power consumption gain due to the use of the optimal deactivation latency $\tau_{d}^{\star}$ against the mean BS vacation time $\bar{\tau}$.

mean $\mathrm{BS}$ vacation time.

In Fig. 5, we plot the power savings gain $\mathcal{P}$ due to the use of the optimal deactivation latency against the mean BS vacation time $\bar{\tau}$. The power savings gain $\mathcal{P}$ is obtained as a ratio of the average power consumption due to the use of the sleep levels defined in Table I to the average power consumption based on the selection of the optimal deactivation latency. We benchmark the result with the case with no sleeping, i.e., the BS retains the no-load power consumption during the vacation period. It can be observed that the selection of the optimal deactivation latency always results in power savings gain since $\mathcal{P}>1, \forall \bar{\tau}$.

\section{CONCLUSiOnS}

In this paper, we have addressed the most energyefficient BS subcomponent deactivation latency while considering that the BS no-load intervals are known in advance by obtaining a closed-form expression of the deactivation latency that minimizes the average BS power consumption. Our analytical insights have revealed that significant power saving can be achieved by selecting the optimal deactivation latency. In addition, further savings can be achieved by addressing the high power consumption during the subcomponent deactivation and reactivation phases.

\section{ACKNOWLEDGEMENT}

We acknowledge the support of EPSRC (GCRF) funds under the grant no. EP/P028764/1.

\section{REFERENCES}

[1] Nokia Siemens Networks, ETSI RRS05-024, 2011.

[2] O. Arnold et al., "Power consumption modeling of different base station types in heterogeneous cellular networks," in Proc. Future Network and Mobile Summit, Florence, Italy, Jun. 2010. 
TABLE I: Discrete Sleep level

\begin{tabular}{|l|l|l|l|l|l|}
\hline \multicolumn{2}{|l|}{$\tau_{i}$} & $\tau_{i}<0.5$ & $0.5<\tau_{i}<1$ & $1<\tau_{i}<2$ & $\tau_{i}>2$ \\
\hline \multirow{3}{*}{ 1-Sleep level } & $\tau_{d i}^{0}(\mathrm{~s})$ & 0 & $\frac{1}{8}$ & $\frac{1}{8}$ & $\frac{1}{8}$ \\
\cline { 2 - 6 } & Power $(\mathrm{W})$ & $P_{0}$ & $P_{0} e^{-\frac{\psi}{8}}$ & $P_{0} e^{-\frac{\psi}{8}}$ & $P_{0} e^{-\frac{\psi}{8}}$ \\
\hline \multirow{2}{*}{ 2-Sleep levels } & $\tau_{d i}^{0}(\mathrm{~s})$ & 0 & $\frac{1}{8}$ & $\frac{1}{4}$ & $\frac{1}{4}$ \\
\cline { 2 - 6 } & Power $(\mathrm{W})$ & $P_{0}$ & $P_{0} e^{-\frac{\psi}{8}}$ & $P_{0} e^{-\frac{\psi}{4}}$ & $P_{0} e^{-\frac{\psi}{4}}$ \\
\hline \multirow{2}{*}{ 3-Sleep levels } & $\tau_{d i}^{0}(\mathrm{~s})$ & 0 & $\frac{1}{8}$ & $\frac{1}{4}$ & $\frac{1}{2}$ \\
\cline { 2 - 6 } & Power $(\mathrm{W})$ & $P_{0}$ & $P_{0} e^{-\frac{\psi}{8}}$ & $P_{0} e^{-\frac{\psi}{4}}$ & $P_{0} e^{-\frac{\psi}{2}}$ \\
\hline
\end{tabular}

[3] G. Auer et al., "How much energy is needed to run a wireless network?" IEEE Wireless Commun. Mag., vol. 18, no. 5, pp. 40-49, Oct. 2011.

[4] B. Debaillie, C. Desset, and F. Louagie, "A flexible and future-proof power model for cellular base stations," in IEEE Vehicular Technology Conference (VTC Spring), 2015.

[5] IMEC, "Power model for today's and future base stations," [Online]. Available: http://www.imec.be/powermodel [Accessed:22 - Feb - 2018]

[6] B. H. Stark, G. D. Szarka, and E. D. Rooke, "Start-up circuit with low minimum operating power for microwatt energy harvesters," IET Circuits, Devices Systems, vol. 5, no. 4, pp. 267-274, Jul 2011.

[7] L. Wang et al., "Small cell switch policy: A consideration of start-up energy cost," in IEEE/CIC International Conference on Communications in China (ICCC), Oct 2014, pp. 231-235.

[8] A. Mohamed et al., "Control-data separation architecture for cellular radio access networks: A survey and outlook," IEEE Communications Surveys \& Tutorials, vol. 18, no. 1, pp. 446465, Firstquarter 2015.

[9] A. Mohamed, O. Onireti, M. A. Imran, A. Imran, and R. Tafazolli, "Correlation-based Adaptive Pilot Pattern in Control/Data
Separation Architecture," in IEEE International Conference on Communications (ICC), Jun 2015.

[10] 3GPP, "TR 38.913: Study on Scenarios and Requirements for Next Generation Access Technologies". Tech. Rep., Release 14 Version 0.4.0. June 2016

[11] P. Lahdekorpi, M. Hronec, P. Jolma, and J. Moilanen, "Energy efficiency of $5 \mathrm{G}$ mobile networks with base station sleep modes," in IEEE Conference on Standards for Communications and Networking (CSCN), Sept 2017, pp. 163-168.

[12] O. Onireti, A. Mohamed, H. Pervaiz, and M. Imran, "Analytical approach to base station sleep mode power consumption and sleep depth," in IEEE International Symposium on Personal, Indoor, and Mobile Radio Communications (PIMRC), Oct.

[13] D. Ferling et al., "D4.3: Final report on green radio technologies,” INFSO-ICT-247733 EARTH, Tech. Rep., Jun 2012.

[14] 3GPP TS 36.104 V10.2.0 (2011-04); LTE Technical Specification (Release 10)

[15] X. Guo et al., "Delay-constrained energy-optimal base station sleeping control," IEEE J. Sel. Areas Commun., vol. 34, no. 5, pp. 1073-1085, May 2016. 